\title{
Characteristics, Outcomes and Predictors of Mortality in Patients Undergoing Transjugular Intrahepatic Portosystemic Shunt
}

\author{
Neta Gotlieb ${ }^{1}$, Jonathan Brill ${ }^{2}$, Oren Shibolet ${ }^{1,2, *}$, Shiri Sherf-Dagan ${ }^{1,3}$ and Isaac Kori ${ }^{4}$ \\ ${ }^{1}$ Liver Unit, The Sackler Faculty of Medicine, Department of Gastroenterology and Liver Diseases, Tel Aviv Medical Center, Tel Aviv, Israel \\ ${ }^{2}$ The Sackler Faculty of Medicine, Tel-Aviv University, Tel Aviv, Israel \\ ${ }^{3}$ Department of Nutrition, Assuta Medical Center, Tel Aviv, Israel \\ ${ }^{4}$ The Sackler Faculty of Medicine, Department of Interventional Radiology, Tel-Aviv Medical Center, Tel Aviv, Israel
}

*Corresponding author: Oren Shibolet, Liver Unit, The Sackler Faculty of Medicine, Department of Gastroenterology and Liver Diseases, Tel Aviv Medical Center, Tel Aviv, Israel, Tel: +972-3-6973984; E-mail: orensh@tlvmc.gov.il

Rec date: June 28, 2018; Acc date: July 16, 2018; Pub date: July 20, 2018

Citation: Gotlieb N, Brill J, Shibolet I, Sherf-Dagan S, Kori I (2018) Characteristics, Outcomes and Predictors of Mortality in Patients Undergoing Transjugular Intrahepatic Portosystemic Shunt. J Clin Gastroenterol Hepatol Vol.2: No.3:15.

\section{Abstract}

Background: Transjugular intrahepatic portosystemic shunt (TIPS) is a procedure for hepatic decompression and lowering of portal hypertension. The procedure is performed angiographically by inserting a metal stent between the hepatic and portal vein. Improved techniques and the expanding indications of TIPS throughout the years have made it more common.

Consequently, a better prediction accuracy of its outcomes and complications is warranted. The aims of this study were to present real-life experience with the TIPS procedure and to identify novel risk factors for shortterm complications and mortality.

Methods and findings: We conducted an observational, retrospective study of all patients who underwent a TIPS procedure from 2005-2017 in a single tertiary center. Data collected from the medical records included demographics, anthropometrics, co-morbidities, biochemical tests, indications and short-term ( $\leq 90$ days) complications. Binary logistic regression analysis was conducted to determine risk factors for 90-day mortality.

Results: A total of 101 patients ( $54 \%$ males and mean age of $55.9 \pm 15.7$ years) underwent technically successful TIPS insertion. The most common complications were hepatic encephalopathy $(43.6 \%, n=44)$ and hepatic decompensation $(17.8 \%, n=18)$. The incidence of 90 -day mortality was $21.8 \%(n=22)$.

Multivariate analysis revealed that older age $(\mathrm{OR}=1.061$, $95 \% \mathrm{Cl}$ : 1.006-1.119), hypertension (OR=6.193, 95\% Cl: 1.666-23.024) and high Model for End-Stage Liver Disease (MELD) score (OR=1.167, 95\% Cl: 1.017-1.329) were independent and significant predictors of early post-TIPS mortality.
Conclusions: Older age, hypertension and high MELD score are independent predictors of early mortality postTIPS. We recommend considering these risk factors when selecting patients for TIPS insertion.

Keywords: Cirrhosis; Portal hypertension; TIPS; Complications; Early mortality; Predictors

Abbreviations: CRF: Chronic Renal Failure; HBV: Hepatitis B Virus; HCC: Hepatocellular Carcinoma; HCV: Hepatitis C Virus; HE: Hepatic Encephalopathy; HVPG: Hepatic VeinPortal Gradient; IHD: Ischemic Heart Disease; MELD: Model for End-Stage Liver Disease; NASH: Non-Alcoholic Steatohepatitis; PTFE: Polytetrafluoroethylene; PVT: Portal Vein Thrombosis; TIPS: Transjugular Intrahepatic Portosystemic Shunt

\section{Introduction}

Portal hypertension is common in patients with cirrhosis [1], its clinical manifestations include variceal bleeding, ascites, splenomegaly with hypersplenism as well as other less common complications such as hepatic hydrothorax, hepatorenal syndrome and others [2].

Transjugular intrahepatic portosystemic shunt (TIPS) is a non-surgical interventional radiology procedure for hepatic decompression and lowering of portal hypertension. TIPS reduces portal pressure by creating an artificial shunt between the portal vein and hepatic vein branches, via insertion of a metal stent [3].

The major indications for TIPS are secondary prevention of variceal bleeding and treatment of refractory ascites [4]. Less commonly, other indications include hepatic hydrothorax, hepatorenal syndrome, Budd-Chiari syndrome and preoperative TIPS for cirrhotic patients undergoing abdominal and pelvic surgery [5]. 
Among the contraindications to the placement of TIPS are severe congestive heart failures, moderate to severe pulmonary hypertension, severe liver failure, and uncontrolled systemic sepsis. Hepatocellular carcinoma (HCC), hepatic encephalopathy (HE) and portal vein thrombosis (PVT) are relative contraindications [4].

The procedure is usually well tolerated and safe, especially when compared to surgical portosystemic shunting [6]. Nevertheless, patients undergoing TIPS can develop significant complications. Technical complications include hepatic vessels laceration and hepatic infarction, hemobilia and arterioportal fistula. Later on, HE is a frequent $(5-50 \%)$ complication in the early post-procedure period $[6,7]$.

Acute deterioration of liver function due to reduced portal venous perfusion [8], as well as shunt stenosis, thrombosis and occlusion may also occur [4]. Early post-procedural mortality ranges from $3 \%$ to $44 \%$ [9], depending on TIPS indication and clinical characteristics of the patient.

Technological innovations such as the use of polytetrafluoroethylene (PTFE) covered stents, reduced the incidence of clinical relapses, stent stenosis, thrombosis and re-interventions and improved outcomes including patients' survival [10].

Existing literature revealed several predictive factors that have been associated with early mortality and poor TIPS outcomes. These include older age, hyperbilirubinemia, renal insufficiency, emergent TIPS and high Model for End-Stage Liver Disease (MELD) score $[9,11,12]$. These prognostic data mandate careful pre-procedural patient selection in order to minimize the risk of short and long-term complications and improve survival rates.

The aims of our study were to assess "real life" experience with the TIPS procedure including indications, complications and outcomes over the past 12 years in a single tertiary center and to identify novel risk factors for early post-TIPS complications and mortality.

\section{Research Methodology}

Our study is a retrospective observational cohort study conducted in a single tertiary center. Patients who underwent TIPS from 2005-2017 were included in the study. Patients under 18 years of age and patients in whom TIPS insertion was technically unsuccessful were excluded. All TIPS procedures were performed at the Tel-Aviv Sourasky Medical Center.

We reviewed medical records to determine age, gender, $\mathrm{BMI}$ ) and clinical data including co-morbidities, underlying causes of cirrhosis and portal hypertension complications. Laboratory parameters at the time of TIPS placement were recorded and included serum bilirubin, creatinine, albumin, sodium, platelet count and international normalized ratio (INR).

MELD score and MELD-Na were calculated using the values for total bilirubin, serum creatinine INR and sodium as previously described [13]. Technical details about the TIPS procedure were also collected including indications, timing (emergent or elective), type of stent used and hepatic veinportal gradient (HVPG) before and after TIPS. The technical aspects of the TIPS procedure were described in detail previously [14].

Short-term ( $\leq 90$ days) complications including technical or stent-related complications, HE (graded according to the WestHaven criteria [15]), renal and hepatic failure as well as TIPS revisions were recorded.

The primary study outcome was early mortality within 90 days following TIPS. Secondary outcomes were the occurrence of TIPS-related early complications and revisions.

\section{Statistical analysis}

Data were analyzed using the statistical program SPSS (Version 24). Continuous variables were presented as means \pm standard deviation (SD) and dichotomous/categorical variables as proportions. Continuous variables that failed the normality test were analyzed using non-parametric tests.

To test differences in continuous variables between 2groups the independent-samples t-test was performed. For comparison of dichotomous or categorical variables the ChiSquare test was performed. Multivariate logistic regression analysis using the stepwise method was conducted in order to determine predictors of early death post-TIPS. The level of significance for all analyses was set at $p<0.05$.

\section{Results}

\section{Study population}

One hundred and one patients (54\% males) met the inclusion criteria during the study period. Pre-TIPS baseline characteristics are presented in Table 1 . The mean age and $\mathrm{BMI}$ at the time of TIPS were $55.9 \pm 15.7$ years and $26.1 \pm 4.5$ $\mathrm{kg} / \mathrm{m}^{2}$, respectively.

The main underlying causes of cirrhosis were hepatitis $C$ virus (HCV) infection (23.8\%, $n=24)$, Budd-Chiari syndrome (17.8\%, n=18), non-alcoholic steatohepatitis (NASH) (17.8\%, $\mathrm{n}=18)$ and cryptogenic cirrhosis $(17.8 \%, \mathrm{n}=18)$, alcoholic hepatitis $(5.9 \%, n=6)$, HBV $(4.9 \%, n=5)$ autoimmune hepatitis $(2.9 \%, n=3)$, sarcoidosis $(2.9 \%, n=3)$, Primary sclerozing cholangitis (PSC) $(2 \%, n=2)$, drug-induced cirrhosis $(2 \%, n=2)$, Wilson disease $(0.9 \%, n=1)$, Schistosomiasis $(1 \%, n=1)$ (Table 1).

Common cirrhotic complications were ascites (73.3\%, $n=74)$, esophageal and gastric varices $(65.4 \%, n=66)$ and hypersplenism (42.6\%, $\mathrm{n}=43$ ). Pre-procedural mean MELD and MELD-Na scores were $11.65 \pm 4.5$ and $15.4 \pm 5.3$, respectively. Main prevalent co-morbidities were Type 2 diabetes $(41.6 \%$, $n=42)$, hypertension $(36.6 \%, n=37)$, ischemic heart disease 
(IHD) $(17.8 \%, n=18)$ and chronic renal failure (CRF) $(16.8 \%$,

$n=17)$.

Table 1 Pre-TIPS baseline characteristics.

\begin{tabular}{|c|c|}
\hline Variables $†$ & All participants $(n=101)$ \\
\hline \multicolumn{2}{|l|}{ Study population } \\
\hline Age (years) & $55.9 \pm 15.7$ \\
\hline Gender (\% male) & 54 \\
\hline \multicolumn{2}{|l|}{ Co-morbidities } \\
\hline BMI $\left(\mathrm{kg} / \mathrm{m}^{2}\right)$ & $26.1 \pm 4.5$ \\
\hline Type 2 Diabetes (\%) & 41.6 \\
\hline Hypertension (\%) & 36.6 \\
\hline Ischemic Heart Disease (\%) & 17.8 \\
\hline Chronic Renal Failure (\%) & 16.8 \\
\hline Cerebrovascular Disease (\%) & 4 \\
\hline \multicolumn{2}{|l|}{ Laboratory Values } \\
\hline INR & $1.3 \pm 0.5$ \\
\hline Serum Total Bilirubin (mg/dL) & $1.2 \pm 0.8$ \\
\hline Serum Albumin (g/dL) & $3.0 \pm 0.6$ \\
\hline Serum Creatinine (mg/dL) & $1.2 \pm 0.5$ \\
\hline Serum Sodium (mEq/L) & $134.9 \pm 5.0$ \\
\hline Platelets (x 109/L) & $167.7 \pm 142.4$ \\
\hline \multicolumn{2}{|l|}{ Clinical characteristics } \\
\hline \multicolumn{2}{|l|}{ Cirrhosis etiology } \\
\hline $\mathrm{HCV}(\%)$ & 23.8 \\
\hline Budd-Chiari syndrome (\%) & 17.8 \\
\hline NASH (\%) & 17.8 \\
\hline Cryptogenic cirrhosis (\%) & 17.8 \\
\hline Others $\ddagger(\%)$ & 22.8 \\
\hline \multicolumn{2}{|l|}{ Cirrhosis complications } \\
\hline Ascites (\%) & 73.3 \\
\hline Varices (\%) & 65.4 \\
\hline Hypersplenism (\%) & 42.6 \\
\hline PVT (\%) & 18.8 \\
\hline Hepatic encephalopathy (\%) & 9.9 \\
\hline $\mathrm{HCC}(\%)$ & 2 \\
\hline MELD score & $11.7 \pm 4.5$ \\
\hline MELD-Na score & $15.4 \pm 5.3$ \\
\hline \multicolumn{2}{|c|}{$\begin{array}{l}\text { Abbreviations: BMI: Body Mass Index, INR: International Normalized Ratio, NASH: Nonalcoholic Steatohepatitis, HCV: Hepatitis C virus, HBV: Hepatitis B virus } \\
\text { PSC: Primary Sclerosing Cholangitis, PVT: Portal vein thrombosis, HCC: Hepatocellular carcinoma, MELD: Model for End-Stage Liver Disease. }\end{array}$} \\
\hline
\end{tabular}




\section{TIPS related data}

TIPS indications and procedure details are shown in Table 2. The major indications for TIPS insertion were refractory ascites $(38.6 \%, n=39)$, secondary prevention of acute or recurrent, esophageal or gastric variceal bleeding $(30.7 \%, n=31)$ and Budd-Chiari syndrome $(18.8 \%, \mathrm{n}=19)$. Less common indications included PVT $(5 \%, n=5)$, hepatic hydrothorax $(3 \%, n=3)$, hepatorenal syndrome $(2 \%, n=2)$ and reduction of portal hypertension prior to major abdominal surgery $(2 \%, n=2)$.

Table 2 TIPS indications and procedure details.

\begin{tabular}{|l|l|}
\hline Indications & Number of patients (\%) \\
\hline Refractory ascites & $39(38.6)$ \\
\hline Variceal bleeding (Acute or refractory) & $31(30.7)$ \\
\hline Budd-Chiari syndrome & $19(18.8)$ \\
\hline Hepatic hydrothorax & $3(3)$ \\
\hline Hepatorenal syndrome & $2(2)$ \\
\hline Others (PVT, preoperative) & $7(6.9)$ \\
\hline Procedure details & $25(24.8)$ \\
\hline Emergent TIPS & $76(75.2)$ \\
\hline Covered stent & $13(12.9)$ \\
\hline Variceal embolization & $19.3 \pm 6.1$ \\
\hline HVPG before shunt-1 & $7.8 \pm 3.1$ \\
\hline HVPG after shunt-1 & $11.5 \pm 5.7$ \\
\hline SHVPG+1 & HVPG: Hepatic vein-portal \\
\hline $\begin{array}{l}\text { Abbreviations: PVT: Portal vein thrombosis, } \\
\text { gradient. }\end{array}$ & \\
\hline †Values expressed as the mean \pm SD & \\
\hline 1Data were available for 94 patients. & \\
\hline
\end{tabular}

The majority of procedures were elective $(75.2 \%, n=76)$ and the others were performed as emergency procedures $(24.8 \%$, $\mathrm{n}=25)$. Seventy-six patients received covered PTFE stent grafts $\left(\right.$ Viatorr $\left.^{\circledR}\right)(75.2 \%)$, whereas the others received bare metal stents $(24.8 \%, n=25)$. Variceal embolization was performed in 13 patients (12.9\%) who had bleeding varices as their TIPS indication. HVPG was measured with a mean pre- and postTIPS pressure of $19.3 \pm 6.1 \mathrm{mmHg}$, and $7.8 \pm 3.1 \mathrm{mmHg}$ respectively.

\section{Complications and mortality}

Adverse events within 90-days of the procedure occurred in $70(69.3 \%)$ of the patients and are summarized in Table 3.

Stent related complications accounted for 25 (24.8\%) of cases, including stent thrombosis ( $n=11,10.9 \%)$, stenosis or occlusion ( $n=13,12.9 \%)$ and a single case of endotipsitis. No events of stent migration were recorded. The most common complication at 90-days post-TIPS was HE, in 44 (43.6\%) patients. Technical access-related complications were the least common, with difficult cannulation $(n=5,5 \%)$, bleeding $(n=3$, $3 \%)$ and puncturing of the liver capsule $(n=2,2 \%)$. Twenty-six TIPS revisions $(26.7 \%)$ were performed within 90-days postTIPS.

TIPS revisions included balloon angioplasty $(n=9)$ and the placement of covered $(n=4)$ and bare metal $(n=4)$ stents within the previous stent. Other procedures such as narrowing or closure of the shunt $(n=3)$, thrombosis evacuation $(n=3)$ and varices embolization without angioplasty or stent placement $(n=3)$. The overall 90 -day patient mortality rate was $21.8 \%$ $(n=22)$ (Table 3). The most common cause of death was liver and/or multi-organ failure $(n=12,54.5 \%)$. Other causes were septic shock $(n=3,13.6 \%)$ and gastrointestinal bleeding $(n=1$, $4.5 \%)$. The remaining causes of death $(n=6,27.3 \%)$ were unspecified.

Table 3 Post-TIPS complications.

\begin{tabular}{|l|l|}
\hline Complications & Number of patients (\%) \\
\hline Difficult cannulation & $5(5)$ \\
\hline Bleeding & $3(3)$ \\
\hline Liver capsule puncture & $2(2)$ \\
\hline Stent thrombosis & $11(10.9)$ \\
\hline Stent stenosis & $13(12.9)$ \\
\hline Endotipsitis & $1(1)$ \\
\hline Hepatic encephalopathy & $29(28.7)$ \\
\hline Grades 1-2 & $15(14.9)$ \\
\hline Grades 3-4 & $44(43.6)$ \\
\hline Total & $15(14.9)$ \\
\hline Renal failure & $18(17.8)$ \\
\hline Hepatic decompensation & $0(0)$ \\
\hline HCC & $1(1)$ \\
\hline Liver transplantation & Abbreviations: HCC: Hepatocellular carcinoma. \\
\hline Death & \\
\hline Ab) & \\
\hline
\end{tabular}

When patients who died 90-days post-TIPS were compared to those who survived, there was no statistical difference noted with regard to the mortality rate of patients with refractory ascites $(45.5 \%$ vs. $36.7 \%, \mathrm{P}=0.352)$ or bleeding varices $(31.8 \%$ vs $39.24 \%, P=0.227)$ as their primary TIPS indication. When TIPS was performed on an emergent basis, the 90 -day mortality rate was $36.4 \%$ ( 8 of 22 ) as compared to a rate of $21.5 \%$ (17 of 79 ) when preformed on an elective basis $(P=0.173)$. While not statistically significant, a trend toward greater mortality rate was observed in emergent TIPS.

\section{Predictors of early death after TIPS placement}

In univariate analysis, older age was significantly associated with death 90-days post-TIPS compared to younger patients (65.2 \pm 10.7 vs. $53.3 \pm 15.9$ years, $P<0.001)$. 
Additionally, a positive history of hypertension, which was defined as previously diagnosed hypertension on medical records and/or previously initiated antihypertensive drug treatment $(72.7 \%$ vs. $26.6 \%, P<0.001)$ and IHD (31.8\% vs. $13.9 \%, \mathrm{P}=0.052$ ) were significantly more common in patients who died 90-days post-TIPS. MELD score, while not reaching statistical significance in univariate analysis showed a trend towards significance with more patients with higher MELD scores died within 90-days post-TIPS (13.1 \pm 6.7 vs $11.3 \pm 3.7$, $\mathrm{P}=0.086)$. MELD-Na score showed a similar trend, with higher scores in patients who died within 90-days post-TIPS $(17 \pm 6.9$ vs $14.9 \pm 4.8, \mathrm{P}=0.103)$.

Binary logistic regression was conducted in order to determine independent predictors of 90-day mortality. To avoid an over-fitted model, we limited the number of examined variables to six, with a rule of thumb of one independent variable for each 15 patients. Age, hypertension and IHD, which were statistically significant on univariate analysis were entered into the analysis. We added MELD score because it showed a trend toward significance and was previously shown to have prognostic value [16]. We also selected the calculated difference of change in HVPG from baseline (Delta HVPG) due to its previously shown relevance in predicting adverse events in cirrhosis [17]. Lastly, we selected gender due to the differences in natural history and outcomes in cirrhosis between men and women [18] and also due to its association with post-TIPS HE, which in turn may contribute to early death rates [7]. Emergent TIPS ( $P=0.154)$, hyperbilirubinemia $(P=0.754)$ and renal insufficiency $(P=0.774)$ were not statistically significant and therefore excluded from this model.

After adjustments for all covariates, increased risk of 90-day mortality was significantly associated with older age, hypertension and higher MELD score (Table 4). Gender, IHD, and Delta HVPG were not found to be associated with increased odds for early death in our cohort.

Table 4 Multivariate analysis for the prediction of post-TIPS 90day mortality.

\begin{tabular}{|l|l|l|}
\hline Variables & OR $(\mathbf{9 5} \% \mathbf{C I})$ & P value \\
\hline Age (years) & $1.061(1.006-1.119)$ & 0.028 \\
\hline Hypertension & $6.193(1.666-23.024)$ & 0.006 \\
\hline MELD score & $1.167(1.017-1.339)$ & 0.028 \\
\hline
\end{tabular}

\section{Discussion}

In the present study, we analyzed a cohort of 101 patients with portal hypertension who underwent the TIPS procedure in a single center over a period of 12 years. The patients had diverse underlying liver diseases and underwent the procedure for various indications. Older age, high MELD score and hypertension were found to be independently associated with increased 90-day mortality.

Our study confirms several risk parameters previously shown to predict mortality in TIPS patients while identifying a novel one. However, we were unable to validate several other factors that were previously shown to predict mortality in these patients. This disparity could be explained in part by the differences in baseline characteristics of the study populations. Our patients had diverse etiologies of cirrhosis with alcoholic liver disease being rare in contrast to previously analyzed populations [8]. Furthermore, because our study covered a later time period than previously reported cohorts, we were able to implement newer clinical practice guidelines [4] which among other recommendations endorsed the use of PTFEcovered stents, further improving the clinical outcomes of the procedure [10].

The MELD score, which was originally developed to predict 3-month survival in TIPS recipients [16], was shown to be superior to Child-Pugh classification in patients undergoing TIPS [12]. Yet, possible limitations of MELD's prognostic value [19] have led to evaluation of other variables which may improve its accuracy, such as serum sodium and the presence of ascites [20]. Our work validates the MELD score as a predictor for mortality following TIPS, showing relatively higher scores in patients who died within 90 days following the procedure, compared to those who survived. The MELD score was assessed as a median value and compared to validated MELD scores $[16,21]$ to establish a cut-off point for increased mortality risk. However, we were unable to define a discrete value from which there was an increased risk for 90-day mortality. Comparison of this study to other publications suggests that a cut-off value for early post-TIPS mortality can vary between populations and indications [22]. Still, it is generally accepted that TIPS should be avoided in MELD scores $>24$, unless it's being used in an emergency setting [16]. Interestingly, our study identifies a population of cirrhotic patients who presented with serious portal hypertension complications with a relatively low MELD score. One plausible explanation could be the impact of persistent ascites, which was the most prevalent TIPS indication in our cohort and of nearly half of the patients who died. Mortality was previously shown to be higher in patients with moderate ascites, with a more prominent effect in lower MELD scores [23]. Prior to TIPS, the major treatment modality of refractory ascites is large-volume paracentesis (LVP) [20]. Repeated LVP can result in the significant caloric loss and induce further catabolic response, even in patients who begin treatment with normal nutritional status [24]. In this cohort, the timing and number of paracenteses, albumin infusions or supplemental feeding prior to TIPS were not reviewed. Hence, we cannot exclude the possibility of these parameters' effects on further postparacentesis circulatory dysfunction, a complication that can have an impact on survival without evident clinical symptoms [25].

We had a large population of NASH-related cirrhotics in our cohort. They may represent a specific population characterized by slow fibrosis progression, low inflammation, preserved synthetic function and high HVPG [26]. Compared with HCVrelated cirrhosis, patients with NASH and low MELD scores ( $\leq$ 15) are less likely to progress, yet are more likely to die or to be withdrawn from the liver transplantation list due to their co-morbidities [27]. This further supports the notion of large 
cirrhotic populations whose poor prognosis is not necessarily reflected by the MELD score.

For these low-MELD patients, other parameters or MELDadjusted models might be better predictors of short-term mortality. MELD-Na, which incorporates serum sodium into its formula, was previously described as a better predictor of death compared to MELD, particularly in patients with low MELD scores [28]. Our results partially supported this theory, as MELD-Na scores, although only showing a trend towards significance, were higher in patients who died in the early period post-TIPS.

Older age in patients with advanced cirrhosis was previously shown to be associated with a higher mortality risk [29]. Similar to previous publications we identified older age as a continuous variable to be a predictor of TIPS mortality. The median age of 60 was assessed as a cut-off in univariate analysis, and patients who were 60 and older had a greater risk of early death post-TIPS, yet this threshold was not significant independently in a multivariate analysis. Previous studies of TIPS in older population cohorts, trying to establish an optimal age cut-off for performing TIPS, have shown inconsistent results. Pan et al. [30] showed an age $\geq 70$ to be associated with poor survival at 90-days and 1 year post-TIPS, while other studies demonstrated that age does not necessarily affect early mortality after the procedure [11,25]. Parvinian et al. found that 90-day mortality was significantly higher in older patients $(\geq 55)$ with intermediate range MELD scores [18-25] undergoing TIPS [31]. This implies that substratification of older patients with lower MELD scores might aid in better patient selection. However, the initial report was underpowered, including 47 patients (with only 23 of them over the age of 55), and did not adjust for other comorbidities.

Age-related mortality has been suggested to reflect deterioration in functioning hepatocyte reserve. However, previous physiological and morphological studies have disputed this suggestion [32,33]. Future studies should focus on other age-associated conditions such as the degree of sarcopenia, or modifiable factors such as the concurrent use of multiple prescription medications.

There has not been much emphasis on medical comorbidities as predictors of survival following TIPS, other than type 2 diabetes mellitus [34] and renal insufficiency [35]. In our cohort IHD was associated with short-term mortality on univariate analysis, but was not independently predictive of death on multivariate analysis. Nonetheless, the interaction between hemodynamic alterations seen post-TIPS [36] and concurrent IHD, may result in worsening of myocardial perfusion, leading to increased morbidity and mortality. In our institute echocardiography is routinely performed as part of a cardiac evaluation prior to TIPS insertion, so our population may be biased towards patients with better myocardial function.

Surprisingly, hypertension was found to be an independent predictor of short-term mortality in our cohort. Arterial hypertension and cirrhosis concurrence is known to be around
7\% [37]. Moreover, these patients may become normotensive as disease progresses due to a decreased overall systemic vascular resistance [38]. Decreased splanchnic resistance and increased peripheral resistance may coexist in some patients, giving rise to increased arterial blood pressure and less pronounced vasodilatory changes [37]. Either way, the insertion of TIPS has been shown to cause worsening of the hyperdynamic circulation of advanced cirrhosis, as demonstrated by increased cardiac output and decreased systemic vascular resistance in the first few months after the procedure [36]. There are major differences in blood pressure regulation between hypertensive and normotensive cirrhosis patients, notably the direct relationship between arterial blood pressure and cardiac output in the former group. Short central circulation time (central blood volume relative to cardiac output) is also substantially reduced in hypertensive cirrhotics and has a significant relation to survival. These mechanisms might be aggravated following TIPS insertion and may explain the increased mortality seen in our patients. On the other hand, the hypertensive cirrhotic patient has less overall peripheral vasodilatation, which may serve as a protecting factor from excessive retention of sodium and water $[39,40]$.

This study's main limitations were its relatively small sample size and retrospective nature, with its built-in weaknesses of data collection and selection bias. Another limitation is the study duration, which spans 12 years in a single center. Patient heterogeneity, variation in selection criteria for TIPS or improvements in medical therapy over the years may have confounded some of our findings. However, our findings represent real life experience and can form the basis of further investigations. Of note, our study was conducted in the era were TIPS indications are expanding, novel techniques are being employed and older and sicker populations are being considered for the procedure. With the data presented here we hope to improve decision-making and identification of atrisk populations.

\section{Conclusion}

In summary, our study identified older age, hypertension and high MELD scores as risk factors for 90-day mortality in patients undergoing TIPS. In addition to current contraindications, further caution should be taken when considering TIPS insertion in these groups of patients. Meticulous patient selection remains paramount for lowering the incidence of complications and associated mortality in patients undergoing TIPS insertion.

\section{References}

1. Bosch J (2007) Vascular deterioration in cirrhosis: the big picture. J Clin Gastroenterol 41: S247-S253.

2. Garcia-Tsao G (2016) Current management of the complications of cirrhosis and portal hypertension: Variceal hemorrhage, ascites, and spontaneous bacterial peritonitis. Dig Dis 34: 382-386. 
3. Boyer TD (2003) Transjugular intrahepatic portosystemic shunt: current status. Gastroenterology 124: 1700-1710.

4. Boyer TD, Haskal ZJ (2010) The role of transjugular intrahepatic portosystemic shunt (TIPS) in the management of portal hypertension: update 2009. Hepatology 51: 306.

5. Smith M, Durham J (2016) Evolving indications for TIPS. Tech Vasc Interv Radiol 19: 36-41.

6. Freedman AM, Sanyal AJ, Tisnado J, Cole PE, Shiffman ML, et al. (1993) Complications of transjugular intrahepatic portosystemic shunt: A comprehensive review. Radiographics 13: 1185-1210.

7. Casadaban LC, Parvinian A, Minocha J, Lakhoo J, Grant CW, et al. (2015) Clearing the confusion over hepatic encephalopathy after TIPS creation: Incidence, prognostic factors, and clinical outcomes. Dig Dis Sci 60: 1059-1066.

8. Casadaban LC, Parvinian A, Couture PM, Minocha J, Knuttinen $M G$, et al. (2014) Characterization of liver function parameter alterations after transjugular intrahepatic portosystemic shunt creation and association with early mortality. American Journal of Roentgenology 203: 1363-1370.

9. Chalasani N, Clark WS, Martin LG, Kamean J, Khan MA, et al. (2000) Determinants of mortality in patients with advanced cirrhosis after transjugular intrahepatic portosystemic shunting. Gastroenterology 118: 138-144.

10. Bureau C, Garcia-Pagan JC, Otal P, Pomier-Layrargues G, Chabbert V, et al. (2004) Improved clinical outcome using polytetrafluoroethylene-coated stents for TIPS: results of a randomized study. Gastroenterology 126: 469-475.

11. Rajan DK, Haskal ZJ, Clark TW (2002) Serum bilirubin and early mortality after transjugular intrahepatic portosystemic shunts: results of a multivariate analysis. J Vasc Interv Radiol 13: 155-161.

12. Salerno F, Merli M, Cazzaniga M, Valeriano $V$, Rossi $P$, et al. (2002) MELD score is better than Child-Pugh score in predicting 3-month survival of patients undergoing transjugular intrahepatic portosystemic shunt. J Hepatol 36: 494-500.

13. Kim WR, Biggins SW, Kremers WK, Wiesner RH, Kamath PS, et al. (2008) Hyponatremia and Mortality among Patients on the Liver-Transplant Waiting List. N Engl J Med 359: 1018-1026.

14. Rossle M, Haag K, Ochs A, Sellinger G, et M, Noldge al. (1994) The transjugular intrahepatic portosystemic stent-shunt procedure for variceal bleeding. N Engl J Med 330: 165-171.

15. Conn HO, Leevy CM, Vlahcevic ZR, Rodgers JB, Maddrey WC, et al. (1977) Comparison of lactulose and neomycin in the treatment of chronic portal-systemic encephalopathy. A double blind controlled trial. Gastroenterology 72: 573-583.

16. Malinchoc M, Kamath PS, Gordon FD, Peine CJ, Rank J, et al. (2000) A model to predict poor survival in patients undergoing transjugular intrahepatic portosystemic shunts. Hepatology 31 : 864-871.

17. Ripoll C, Banares R, Rincon D, Catalina MV, Lo lacono O, et al. (2005) Influence of hepatic venous pressure gradient on the prediction of survival of patients with cirrhosis in the MELD Era. Hepatology 42: 793-801.

18. Guy J, Peters MG (2013) Liver disease in women: The influence of gender on epidemiology, natural history, and patient outcomes. Gastroenterol Hepatol (N Y) 9: 633-639.

19. Freeman RB (2005) MELD: the holy grail of organ allocation? J Hepatol 42: 16-20.
20. Heuman DM, Abou-Assi SG, Habib A, Williams LM, Stravitz RT, et al. (2004) Persistent ascites and low serum sodium identify patients with cirrhosis and low MELD scores who are at high risk for early death. Hepatology 40: 802-810.

21. Russo MW, Jacques PF, Mauro M, Odell P, Brown Jr RS (2002) Predictors of mortality and stenosis after transjugular intrahepatic portosystemic shunt. Liver Transpl 8: 271-277.

22. Al Sibae MR, Cappell MS (2011) Accuracy of MELD scores in predicting mortality in decompensated cirrhosis from variceal bleeding, hepatorenal syndrome, alcoholic hepatitis, or acute liver failure as well as mortality after non-transplant surgery or TIPS. Dig Dis Sci 56: 977-987.

23. Somsouk M, Kornfield R, Vittinghoff E, Inadomi JM, Biggins SW (2011) Moderate ascites identifies patients with low MELD awaiting liver transplantation with high mortality risk. Liver transplantation: Official publication of the American Association for the Study of Liver Diseases and the International Liver Transplantation Society 17: 129-136.

24. Sorrentino P, Castaldo G, Tarantino L, Bracigliano A, Perrella A, et al. (2012) Preservation of nutritional-status in patients with refractory ascites due to hepatic cirrhosis who are undergoing repeated paracentesis. J Gastroenterol Hepatol 27: 813-822.

25. Harrod-Kim P, Saad W, Waldman D (2006) Predictors of early mortality after transjugular intrahepatic portosystemic shunt creation for the treatment of refractory ascites. J Vasc Interv Radiol 17: 1605-1610.

26. Calzadilla Bertot L, Adams LA (2016) The natural course of nonalcoholic fatty liver disease. Int J Mol Sci 17: 774.

27. O'Leary JG, Landaverde C, Jennings L, Goldstein RM, Davis GL (2011) Patients with NASH and cryptogenic cirrhosis are less likely than those with hepatitis $C$ to receive liver transplants. Clinical gastroenterology and hepatology: The Official Clinical Practice Journal of the American Gastroenterological Association 9: 700-704.

28. Kwong AJ, Lai JC, Dodge JL, Roberts JP (2015) Outcomes for liver transplant candidates listed with low MELD. liver transplantation: Official publication of the American Association for the Study of Liver Diseases and the International Liver Transplantation Society 21: 1403-1409.

29. Schlichting $P$, Christensen E, Andersen PK, Fauerholdt L, Juhl E, et al. (1983) Prognostic factors in cirrhosis identified by Cox's regression model. Hepatology 3: 889-895.

30. Pan JJ, Chen C, Caridi JG, Geller B, Firpi R, et al. (2008) Factors predicting survival after transjugular intrahepatic portosystemic shunt creation: A 15 years' experience from a single tertiary medical center. J Vasc Interv Radiol 19: 1576-1581.

31. Parvinian A, Shah KD, Couture PM, Minocha J, Knuttinen MG, et al. (2013) Older patient age may predict early mortality after transjugular intrahepatic portosystemic shunt creation in individuals at intermediate risk. J Vasc Interv Radiol 24: 941-946.

32. Wakabayashi $H$, Nishiyama $Y$, Ushiyama T, Maeba T, Maeta $H$ (2002) Evaluation of the effect of age on functioning hepatocyte mass and liver blood flow using liver scintigraphy in preoperative estimations for surgical patients: Comparison with CT volumetry. J Surg Res 106: 246-253.

33. Schmucker DL (1998) Aging and the liver: An update. J Gerontol A Biol Sci Med Sci 53: B315-B320.

34. Shah SH, Lui HF, Lui HF, Helmy A, Redhead DN, et al. (2001) Transjugular intrahepatic portosystemic stent-shunt insufficiency 
and the role of diabetes mellitus. Eur J Gastroenterol Hepatol 13: $257-261$.

35. Anderson CL, Saad WE, Kalagher SD, Caldwell S, Sabri S, et al. (2010) Effect of transjugular intrahepatic portosystemic shunt placement on renal function: A 7-year, single-center experience. J Vasc Interv Radiol 21: 1370-1376.

36. Lotterer E, Wengert A, Fleig WE (1999) Transjugular intrahepatic portosystemic shunt: Short-term and long-term effects on hepatic and systemic hemodynamics in patients with Cirrhosis. Hepatology 29: 632-639.

37. Henriksen JH, Moller S (2006) Liver cirrhosis and arterial hypertension. World Journal of Gastroenterology WJG 12: 678-685.
38. Loyke HF (1962) Reduction of hypertension after liver disease. Arch Intern Med 110: 45-49.

39. Henriksen JH, Fuglsang S, Bendtsen F, Møller S (2006) Arterial hypertension in cirrhosis: arterial compliance, volume distribution, and central haemodynamics. Gut 55: 380.

40. Moller S, Bendtsen F, Christensen E, Henriksen JH (1995) Prediction of bleeding risks and death in cirrhosis based on hemodynamic and metabolic variables. A study of patients with esophageal varices without previous bleeding. Ugeskr Laeger 157: 5389-5393. 